\title{
Giant Magnetoresistance in an all-oxide spacerless junction
}

\author{
M. P. Singh, B. Carvello, and L. Rannd* \\ Laboratoire Louis Néel, CNRS-UJF-INPG, Polygone CNRS, \\ BP 166, 38042 Grenoble cedex 09, France
}

(Dated: 10 July 2006)

\begin{abstract}
We report the fabrication of an oxide-specific type of magnetoresistive junction, which is a ferromagnetic bilayer. Both electrodes are high spin-polarization oxides: magnetite $\left(\mathrm{Fe}_{3} \mathrm{O}_{4}\right)$ and manganite $\left(\mathrm{La}_{0.7} \mathrm{Sr}_{0.3} \mathrm{MnO}_{3}\right)$. Negligible magnetic coupling between both ferromagnetic electrodes is realised, which allows to obtain parallel and antiparallel magnetic configurations of the electrodes when sweeping the applied magnetic field. The structure exhibits negative giant magnetoresistance (GMR) at low temperatures. This negative MR shows that both electrodes stay spin-polarized at the interface and have opposite spin polarizations, i.e. the $\mathrm{Fe}_{3} \mathrm{O}_{4}$ layer has a negative spin polarization at low temperature. Maximum GMR (-5\%) is obtained at $55 \mathrm{~K}$.
\end{abstract}

PACS numbers: 73.40.-c, 75.47.Lx,75.70.Cn (Transport through interfaces, Manganites, Magnetic Properties of interfaces )

\section{INTRODUCTION}

Highly spin-polarized ferromagnetic oxides, such as $\mathrm{CrO}_{2}, \mathrm{Fe}_{3} \mathrm{O}_{4}, \mathrm{La}_{0.7} \mathrm{Sr}_{0.3} \mathrm{MnO}_{3}$ (LSMO) have been the focus of recent fundamental and technological studies in the field of spin electronics. Using these materials, various devices, such as giant magnetoresistance (GMR) junctions 1 and tunnel magnetoresistance (TMR) junctions [2] have been fabricated and studied. To fabricate a magnetoresistive device based on a junction, usually two ferromagnetic layers are separated by a thin non magnetic layer (the spacer). The nature of the spacer is chosen in order to control the spin-dependent transport mechanism at the interface: metallic spacer (GMR) or insulating spacer (TMR).

In the usual case of transition metal electrodes, the thickness of the spacer is chosen in order to magnetically decouple the magnetic layers (i.e. thickness larger than a few atomic planes to break the direct coupling exchange path, and to prevent indirect coupling such as the RKKY one). The transport across the spacer must conserve the spin information, thus the spacer thickness must be kept thinner than a few mean free paths (current-in-plane cipGMR) or spin diffusion lengths (current-perpendicularto-plane cpp-GMR) or a few $1 / \mathrm{k}_{\mathrm{F}}$ (tunnel probability in TMR junction).

A spacer is not necessary if it is possible to weaken the magnetic coupling between both electrodes. Such devices have already been proposed: ballistic junctions [3] or break junctions [4]. These junctions were designed to break the exchange coupling between two transition metals. To achieve this, both electrodes have to be mechanically separated, which is a difficult step, source of non reproducibilities, and sensitive to parasitic phenomena such as magnetostriction [5]. In this paper we pro-

*Electronic address: laurent.ranno@grenoble.cnrs.fr pose a simple solid state structure, adapted to collective fabrication.

In oxides, magnetic coupling is due to indirect exchange (3d ion - oxygen - $3 \mathrm{~d}$ ion) and it is very sensitive to the atomic details of such a bond. For example it is possible to weaken the coupling by changing the bond angle (manganite's $\mathrm{T}_{\mathrm{C}}$ varies as a function of the Mn-O-Mn bond angle $6 \mid$ ). Thus, tuning the interfacial magnetic coupling is achievable in oxides.

The spin-polarization of a material is positive if the majority spin at the Fermi level is parallel to the magnetization and negative if the minority spin at the Fermi level is parallel to the magnetization. Half metallic ferromagnets have a spin polarization of $100 \%$ (only one spin direction is present at the Fermi level). Magnetite $\left(\mathrm{Fe}_{3} \mathrm{O}_{4}\right)$ stands out as a predicted half metallic ferromagnet (ferrimagnet in fact) with negative spin polarization 7] and a remarkably high Curie temperature $\left(\mathrm{T}_{\mathrm{C}}\right)$ of $858 \mathrm{~K} . \mathrm{La}_{0.7} \mathrm{Sr}_{0.3} \mathrm{MnO}_{3}$ is predicted to have $100 \%$ positive polarization [8] with $\mathrm{T}_{\mathrm{C}}$ of $350 \mathrm{~K}$. A junction between two such half metallic ferromagnetic compounds would in theory behave as an ideal magnetic-field-controlled switch with $100 \%$ negative magnetoresistance (MR).

As mentioned above, recent efforts have been made to fabricate $\mathrm{Fe}_{3} \mathrm{O}_{4} / \mathrm{I} / \mathrm{La}_{0.7} \mathrm{Sr}_{0.3} \mathrm{MnO}_{3}$ junctions, where $\mathrm{I}$ is $\mathrm{SrTiO}_{3}, \mathrm{CoCr}_{2} \mathrm{O}_{4}$ [9, 10], but a $\mathrm{Fe}_{3} \mathrm{O}_{4} / \mathrm{La}_{0.7} \mathrm{Sr}_{0.3} \mathrm{MnO}_{3}$ junction without a spacer has never been proposed.

\section{EXPERIMENTAL DETAILS}

The $\mathrm{Fe}_{3} \mathrm{O}_{4} / \mathrm{La}_{0.7} \mathrm{Sr}_{0.3} \mathrm{MnO}_{3}$ bilayers were grown on (001)-oriented $\mathrm{SrTiO}_{3}$ (STO) substrates using pulsed laser deposition. First, the LSMO layer was grown at $1023 \mathrm{~K}$ under $40 \mathrm{~Pa}$ of $\mathrm{O}_{2}$, then the $\mathrm{Fe}_{3} \mathrm{O}_{4}$ layer was grown at $623 \mathrm{~K}$ under $5.10^{-4} \mathrm{~Pa}$ of $\mathrm{O}_{2}$. The thickness of LSMO was $50 \mathrm{~nm}$ whereas the $\mathrm{Fe}_{3} \mathrm{O}_{4}$ was grown with two different thicknesses, $15 \mathrm{~nm}$ and $50 \mathrm{~nm}$, estimated in situ by optical reflectometry. Prior to the deposition, 
the substrate was heated in oxygen up to the deposition temperature.

To study the magnetotransport properties of the junction, $50 \mathrm{~nm} \mathrm{Au}$ was deposited upon the 15/50 nm $\mathrm{Fe}_{3} \mathrm{O}_{4} / \mathrm{LSMO} / \mathrm{STO}$ structure at room temperature using the sputtering technique and subsequently, junctions of $500 \times 500 \mu \mathrm{m}$ and $140 \times 140 \mu \mathrm{m}$ were fabricated by photolithography and Ar ion etching process. All transport measurements were carried out with cpp geometry and applied magnetic field parallel to the plane.

X-ray diffraction (XRD) study was carried out to examine the structural properties of the bilayers. Despite the $1 \%$ lattice mismatch between LSMO and STO, the LSMO growth is pseudomorphic up to a critical thickness $(100 \mathrm{~nm})$ larger than the thickness chosen for these bilayers [1]]. The LSMO film is epitaxially-strained $\left(0.18^{\circ}\right.$ FWHM rocking-curve) and a large epitaxially-induced magneto-elastic anisotropy is present 11]. The $\mathrm{Fe}_{3} \mathrm{O}_{4}$ film on LSMO is textured with multiple orientations (diffraction peaks corresponding to [001] and [011] directions, but not [311]), whereas $\mathrm{Fe}_{3} \mathrm{O}_{4}$ films on $\mathrm{SrTiO}_{3}$ deposited under similar conditions, were grown textured along the [001] direction with $1^{\circ}$ FWHM rocking curve. The details of the deposition of films of LSMO and $\mathrm{Fe}_{3} \mathrm{O}_{4}$ on STO have been reported elsewhere [11, 12].

\section{RESULTS}

To check for magnetic coupling between both oxide layers, $\mathrm{M}(\mathrm{H})$ hysteresis loops of the unpatterned 50/50 nm bilayer structure were measured in the temperature range 10-350 $\mathrm{K}$ and up to 3 Tesla using a VSM and a SQUID magnetometer.

Fig. 1 shows the typical hysteresis loop from an unpatterned bilayer structure at $50 \mathrm{~K}$, with the magnetic field applied along the substrate [110] axis, which is the easy direction of the $\mathrm{La}_{0.7} \mathrm{Sr}_{0.3} \mathrm{MnO}_{3}$ layer. The hysteresis loop clearly shows two distinct coercive fields (about $5 \mathrm{mT}$ and $100 \mathrm{mT}$ ), which correspond to LSMO and $\mathrm{Fe}_{3} \mathrm{O}_{4}$, respectively. The temperature dependence of $\mathrm{Fe}_{3} \mathrm{O}_{4}$ coercivity shows the Verwey transition at $110 \mathrm{~K}$ $\left(\mathrm{T}_{\mathrm{V}}=122 \mathrm{~K}\right.$ in single crystals $)$.

To study the magnetic coupling between these layers, minor loops of the softer layer (LSMO) were measured at $10 \mathrm{~K}$. No shift of the loops was detected. Thus no exchange bias field larger than $3 \mathrm{mT}$ exists. The large coercivity difference between layers and squareness of the LSMO hysteresis loop create well-defined parallel and antiparallel magnetic configurations.

As far as transport is concerned, we have measured $\mathrm{I}(\mathrm{V})$ characteristics from 5 to $300 \mathrm{~K}$. They are linear up to the point where heating effects come into play. The evolution of the resistance with temperature (Fig. 2) can be divided into 3 regimes. The high temperature regime, above $90 \mathrm{~K}$, exhibits the well-known resistance and CMR (colossal magnetoresistance) of manganites, and can thus be attributed to the LSMO electrode, which dominates the transport at these temperatures due to geometrical reasons. Between 30 and $90 \mathrm{~K}$, the transport is thermally activated, due to the increasing $\mathrm{Fe}_{3} \mathrm{O}_{4}$ dominance when temperature decreases. Below $30 \mathrm{~K}$, a plateau is observed, which is surprising in a $\mathrm{Fe}_{3} \mathrm{O}_{4}$-dominated regime. This remains to be investigated, but could be explained by the onset of a hot electron transport mechanism due to the high electric field $(40 \mathrm{kV} / \mathrm{cm})$, such as the one observed in [13].

At high field (1 to $6 \mathrm{~T}$ range), the junction shows a negative magnetoresistance. This high field $\mathrm{MR}$ is large below $40 \mathrm{~K}$ (over $-1 \% / \mathrm{T}$, consistent with $\mathrm{Fe}_{3} \mathrm{O}_{4}$ thin films), smaller between 40 and $100 \mathrm{~K}$, and increases to high values $(-2 \% / \mathrm{T})$ at high temperature (LSMO CMR). In the intermediate regime, though, this negative slope is only visible above $2 \mathrm{~T}$. Under that field, $\mathrm{Fe}_{3} \mathrm{O}_{4}$ is poorly saturated, and GMR dominates (see below), giving a positive slope.

In the intermediate temperature range, the magnetic field dependent transport measurement shows a characteristic inverse GMR behavior (Fig. 3, the applied field is sweeped as the arrows indicate). The magnetic fields at which the junction resistance changes the most abruptly correspond to the coercivities of both oxide layers and the junction resistance is lower when the magnetizations of both layers are antiparallel to each other. The GMR was measured at $\pm 100 \mu \mathrm{A}$ constant current as a function of temperature (Fig. 2). $R_{\uparrow \downarrow}$ is measured at $20 \mathrm{mT}$ and $R_{\uparrow \uparrow}$ at $400 \mathrm{mT}$. This magnetoresistance diminishes in absolute value both above $90 \mathrm{~K}$ and below $30 \mathrm{~K}$. The maximum GMR of $-5.2 \%$ is found at $55 \mathrm{~K}$.

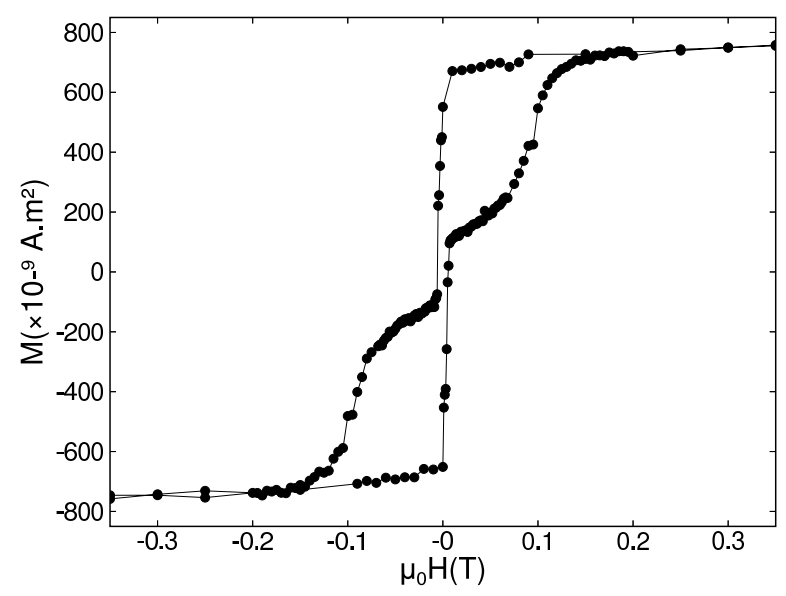

FIG. 1: Hysteresis loops of unpatterned bilayer film at $50 \mathrm{~K}$ 


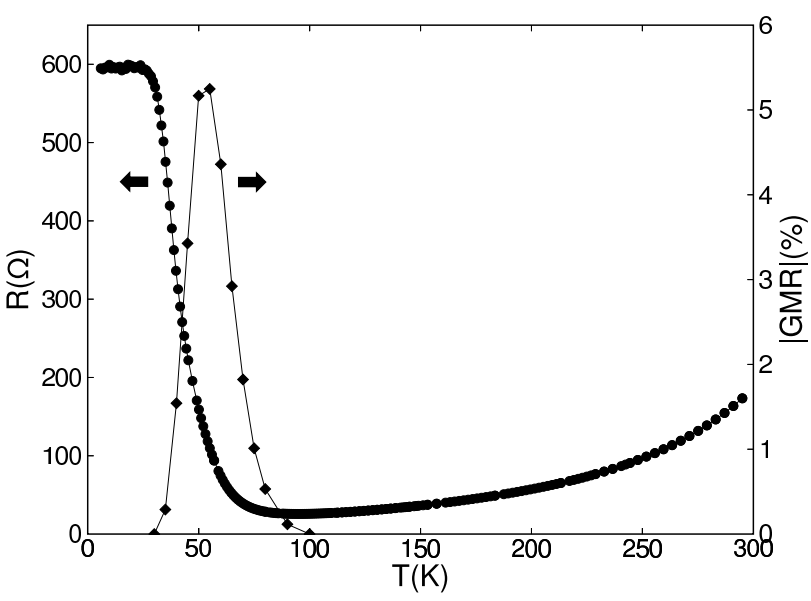

FIG. 2: Resistance and GMR of a $140 \times 140 \mu \mathrm{m}$ junction measured with a current of $100 \mu \mathrm{A}$



FIG. 3: $\mathrm{R}(\mathrm{H})$ magnetotransport measurement of a $140 \times 140$ $\mu \mathrm{m}$ junction for $\mathrm{T}=55 \mathrm{~K}$

\section{DISCUSSION}

The two keypoints which have to be discussed are the origin of the magnetic decoupling of the $\mathrm{La}_{0.7} \mathrm{Sr}_{0.3} \mathrm{MnO}_{3}$ and $\mathrm{Fe}_{3} \mathrm{O}_{4}$ electrodes and the transport mechanism responsible for the large magnetoresistance. The ferromagnetic coupling mechanism is double exchange $\left(\mathrm{Mn}^{3+}-\mathrm{O}-\right.$ $\mathrm{Mn}^{4+}$ bonds) in the case of LSMO, and is superexchange $\left(\mathrm{Fe}^{3+}(\mathrm{A})-\mathrm{O}-\mathrm{Fe}^{2+/ 3+}(\mathrm{B})\right.$ bonds) as well as double exchange $\left(\mathrm{Fe}^{3+}(\mathrm{B})-\mathrm{O}-\mathrm{Fe}^{2+}(\mathrm{B})\right.$ bonds) in the case of $\mathrm{Fe}_{3} \mathrm{O}_{4}$. To magnetically decouple the two layers, these nearestneighbour mechanisms have to be weakened. The interface between LSMO and $\mathrm{Fe}_{3} \mathrm{O}_{4}$ is a structurally disordered layer due to the $6.7 \%$ lattice mismatch, which prevents heteroepitaxy. In oxides, due to the localised character of electrons, weakening the exchange is much easier than in transition metals where electrons are more delocalised (RKKY coupling range can reach a few nanometers). So one disordered layer due to the lattice mismatch between a perovskite and a spinel ferromagnet is enough to reduce the exchange coupling and to decouple both layers. This is a general statement since the lattice mismatch between a spinel and a perovskite structure will always be a few $\%$.

We claim that our structure is a bilayer. However the presence of an intermixed layer between the electrodes has to be ruled out to support this claim. In our system any intermixing layer would be made of $\mathrm{Fe}$ and $\mathrm{Mn}$ ions and therefore it would be magnetic. Since transport in conducting ferromagnetic oxides is based on a nearest-neighbour hopping mechanism, any magnetic layer depolarizes the current. Thus, the characteristic type of magnetoresistance we can measure rules out the presence of an intermixing layer. Furthermore since the $\mathrm{Fe}_{3} \mathrm{O}_{4}$ layer is deposited at low temperature $(623 \mathrm{~K})$, the spinel/perovskite interface is expected to be stable. We have recently conducted a TEM study on the $\mathrm{SrTiO}_{3} / \mathrm{Fe}_{3} \mathrm{O}_{4}$ interface, which showed that the perovskite / spinel mismatch can be accomodated through a regular array of dislocations [14] and confirms that no intermixing takes place.

As the MR is small (-5\%), spin disorder in the interfacial plane, leading to a partial depolarisation, cannot be ruled out. However, this cannot be called a distinct magnetic layer, it is better characterized as an interfacial disorder.

The two electrodes are in direct electrical contact, so the nature of the transport mechanism is related to the presence or absence of an electrical barrier. The cpp transport characteristics are ohmic, and the $\mathrm{R}(\mathrm{T})$ exhibits no regime that could be interpreted as a tunnel transport.

Since a significant MR has been measured and the characteristic fields of this $\mathrm{MR}$ are the coercive fields of both electrodes a spin-coherent mechanism has to be proposed. Through the interface, transport could still be based on a hopping mechanism. Interface disorder only reduces hopping integrals (also impacting magnetism by 
reducing the double-exchange coupling). Thus the MR mechanism is closer to the cpp-GMR than to the TMR mechanism and the only significant interfacial resistance is the GMR itself.

The value of the magnetoresistance is difficult to interpret in a quantitative manner, given that the resistance of the interface is not dominant compared to that of the electrodes. Thus the $-5.2 \%$ GMR ratio is not intrinsic, and could be enhanced through an optimized junction pattern.

It is also worth noting that it is difficult to obtain a real parallel or antiparallel state with $\mathrm{Fe}_{3} \mathrm{O}_{4}$. Because of structural defects present in all $\mathrm{Fe}_{3} \mathrm{O}_{4}$ thin films (antiphase boundaries), the remanence is less than $80 \%$, and full saturation is not achieved even at large fields. Lastly, the decay of the GMR at high temperature can be explained by the decrease of LSMO polarisation well below
$\mathrm{T}_{\mathrm{C}}$, as is known from other studies $([15])$. Nonetheless, our measurements evidence negative spin polarization of $\mathrm{Fe}_{3} \mathrm{O}_{4}$, as predicted, down to the interface with LSMO.

For these reasons, we propose that the mechanism responsible for the exchange weakening and the large MR in our bilayer is fundamentally different from the TMR and spacer-assisted cpp-GMR mecanisms reported in other studies. It is purely interfacial (i.e. spacerless) and specific to oxides.

\section{Acknowledgments}

One of the authors (MPS) would like to thank EGIDE and the French Foreign Ministry, for providing the postdoctoral fellowship.
[1] S. van Dijken, X. Fain, S. M. Watts, and J. M. D. Coey, Phys. Rev. B 70 70, 052409 (2004).

[2] P. Seneor, A. Fert, J.-L. Maurice, F. Montaigne, F. Petroff, and A. Vaurès, Appl. Phys. Lett. 74, 4017 (1999).

[3] N. Garcia, M. Muñoz, and Y.-W. Zhao, Phys. Rev. Lett. 82, 2923 (1999).

[4] M. Gabureac, M. Viret, F. Ott, and C. Fermon, Phys. Rev. B 69, 100401(R) (2004).

[5] W. F. Egelhoff, L. Gan, H. Ettedgui, Y. Kadmon, C. J. Powell, P. J. Chen, A. J. Shapiro, R. D. McMichael, J. J. Mallett, T. P. Moffat, M. D. Stiles, and E. B. Svedberg, J. Appl. Phys. 95, 7554 (2004).

[6] H. Y. Hwang, S.-W. Cheong, P. G. Radaelli, M. Marezio, and B. Batlogg, Phys. Rev. Lett. 75, 914 (1995).

[7] A. Yanase and K. Siratori, J. Phys. Soc. Jap. 53, 312 (1984).
[8] E. A. Livesay, R. N. West, S. B. Dugdale, G. Santi, and T. Jarlborg, J. Phys.: Condens Matter 11, L279 (1999).

[9] S. B. Ogale, K. Ghosh, S. P. Pai, M. Robson, E. Li, I. Jin, R. L. Greene, R. Ramesh, T. Venketesan, and M. Johnson, Mater. Sci. Eng. B 56, 134 (1998).

[10] G. Hu, R. Chopdekar, and Y. Suzuki, J. Appl. Phys. 93, 7516 (2003).

[11] L. Ranno, A. Llobet, R. Tiron, and E. Favre-Nicolin, Appl. Surf. Sc. 188, 170 (2002).

[12] B. Carvello and L. Ranno, J. Magn. Magn. Mater. 272276, 1926 (2004).

[13] G. Chern, S. D. Berry, D. M. Lind, H. Mathias, and L. R. Testardi, Phys. Rev. B 45, 3644 (1992).

[14] B. Carvello, P. Warin, and L. Ranno, to be published.

[15] E. Favre-Nicolin, L. Ranno, C. Dubourdieu, and M. Rosina, Thin Solid Films 400, 165 (2001). 